\title{
Performance of Eigenvalue-based Signal Detectors with Known and Unknown Noise Level
}

\author{
Boaz Nadler \\ Weizmann Institute of Science, Israel \\ boaz.nadler@weizmann.ac.il
}

\author{
Federico Penna \\ Politecnico di Torino, Italy \\ federico.penna@polito.it
}

\author{
Roberto Garello \\ Politecnico di Torino, Italy \\ garello@polito.it
}

\begin{abstract}
In this paper we consider signal detection in cognitive radio networks, under a non-parametric, multi-sensor detection scenario, and compare the cases of known and unknown noise level. The analysis is focused on two eigenvalue-based methods, namely Roy's largest root test, which requires knowledge of the noise variance, and the generalized likelihood ratio test, which can be interpreted as a test of the largest eigenvalue vs. a maximum-likelihood estimate of the noise variance. The detection performance of the two considered methods is expressed by closed-form analytical formulas, shown to be accurate even for small number of sensors and samples. We then derive an expression of the gap between the two detectors in terms of the signal-to-noise ratio of the signal to be detected, and we identify critical settings where this gap is significant (e.g., small number of sensors and signal strength). Our results thus provide a measure of the impact of noise level knowledge and highlight the importance of accurate noise estimation.
\end{abstract}

\section{INTRODUCTION}

Spectrum sensing is a central issue in cognitive radio (CR) systems [1]-[3] and has attracted great research interest in the last decade. In particular, sensing techniques based on the eigenvalues of the received sample covariance matrix (see, for instance, [4]-[10]) recently emerged as a promising solution, as they do not require a priori assumptions on the signal to be detected, and typically outperform the popular energy detection method when multiple sensors are available. Eigenvaluebased detection (EBD) schemes can be further divided into two categories: methods that assume knowledge of noise level (referred to as "semi-blind" [4]), and methods that do not assume this knowledge ("blind"). Methods belonging to the first class provide better performance when the noise variance is known exactly, whereas blind methods are more robust to uncertain or varying noise level.

In this paper, we analyze the performance of two nearlyoptimal $^{1}$ detection criteria in their respective categories: for known noise variance, the largest eigenvalue test, or Roy's largest root test (RLRT), originally proposed in [11] and introduced in CR by [9] (derived as a "blindly combined energy detector"); for unknown noise variance, the test of the largest eigenvalue divided by trace of the covariance matrix, introduced in CR by [7] as a generalized likelihood ratio test (GLRT) and previously appeared in signal processing [12] and statistics literature [13], [14]. The main contributions of our analysis are: (i) derivation of novel expressions for the

\footnotetext{
${ }^{1}$ See Sec. III for a more rigorous definition.
}

false-alarm and detection probabilities of the GLRT, accurate for finite numbers of samples and receivers; (ii) analytical expression of the performance gap between the two detectors. Based on these results, we show that in some scenarios (large number of sensors, relatively high signal-to-noise ratio) the performance improvement of RLRT over GLRT is marginal, whereas in other settings (few sensors, low signal-to-noise ratio) the gap is significant. In other words, exact knowledge of the noise level may result, in some cases, in an increased detection capability of the order of several $\mathrm{dBs}$.

The paper is organized as follows: the signal model is introduced in Sec. II; the considered detection methods are presented in Sec. III; a comparative performance analysis of RLRT and GLRT is provided in Sec. IV; the formulas derived analytically are validated by numerical simulations in Sec. V; Sec. VI concludes the paper.

\section{MODEL}

We consider a multi-sensor detection setting, where the detector constructs its test statistic from $K$ sensors (receivers or antennas) and $N$ time samples. Let $\boldsymbol{y}(n)=\left[y_{1}(n) \ldots y_{K}(n)\right]^{T}$ be the $K \times 1$ received vector at time $n$, where the element $y_{k}(n)$ is the discrete baseband complex sample at receiver $k$.

Under $\mathcal{H}_{0}$, the received vector consists of $K$ complex Gaussian noise samples with zero mean and variance $\sigma_{v}^{2}$

$$
\left.\boldsymbol{y}(n)\right|_{\mathcal{H}_{0}}=\boldsymbol{v}(n)
$$

where $\boldsymbol{v}(n) \sim \mathcal{N}_{\mathbb{C}}\left(\mathbf{0}_{K \times 1}, \sigma_{v}^{2} \boldsymbol{I}_{K \times K}\right)$. Under $\mathcal{H}_{1}$, in contrast, the received vector contains signal plus noise

$$
\left.\boldsymbol{y}(n)\right|_{\mathcal{H}_{1}}=\boldsymbol{x}(n)+\boldsymbol{v}(n)=\boldsymbol{h} s(n)+\boldsymbol{v}(n)
$$

where $s(n)$ is the transmitted signal sample, modeled as a Gaussian $^{2}$ random variable with zero mean and variance $\sigma_{s}^{2}$, and $\boldsymbol{h}$ is the $K \times 1$ unknown complex channel vector. The channel is assumed to be memoryless and constant during the detection time. Under $\mathcal{H}_{1}$, we define the SNR at the receiver as

$$
\rho \triangleq \frac{\mathrm{E}\|\boldsymbol{x}(n)\|^{2}}{\mathrm{E}\|\boldsymbol{v}(n)\|^{2}}=\frac{\sigma_{s}^{2}}{\sigma_{v}^{2}} \frac{\|\boldsymbol{h}\|^{2}}{K},
$$

where $\|\cdot\|$ denotes Euclidean $\left(L_{2}\right)$ norm.

\footnotetext{
${ }^{2}$ The Gaussian signal assumption simplifies the mathematical analysis and, as far as detection performance is concerned, turns out to be a reasonable approximation also for digitally modulated signals (e.g., 4/8-PSK, 16-QAM etc.) after pulse-shape filtering and non-coherent sampling.
} 
The received samples are stored by the detector in the $K \times N$ matrix

$$
\boldsymbol{Y} \triangleq[\boldsymbol{y}(1) \ldots \boldsymbol{y}(N)]=\boldsymbol{h} \boldsymbol{s}+\boldsymbol{V}
$$

where $s \triangleq[s(1) \ldots s(N)]$ is a $1 \times N$ signal vector and $\boldsymbol{V} \triangleq[\boldsymbol{v}(1) \ldots \boldsymbol{v}(N)]$ is a $K \times N$ noise matrix. The sample covariance matrix $\boldsymbol{R}$ is then defined as

$$
\boldsymbol{R} \triangleq \frac{1}{N} \boldsymbol{Y} \boldsymbol{Y}^{H}
$$

Let $\lambda_{1} \geq \ldots \geq \lambda_{K}$ be the eigenvalues of $\boldsymbol{R}$ (without loss of generality, sorted in decreasing order).

In general, let $T$ be the test statistic employed by the detector to distinguish between $\mathcal{H}_{0}$ and $\mathcal{H}_{1}$ : several possible test statistics will be examined throughout the paper. To make the decision, the detector compares $T$ against a pre-defined threshold $t$ : if $T>t$ it decides for $\mathcal{H}_{1}$, otherwise for $\mathcal{H}_{0}$. As a consequence, the probability of false alarm is defined as

$$
P_{\text {fa }}=\operatorname{Pr}\left(T>t \mid \mathcal{H}_{0}\right)
$$

and the probability of detection as

$$
P_{\mathrm{d}}=\operatorname{Pr}\left(T>t \mid \mathcal{H}_{1}\right) .
$$

Usually, the decision threshold $t$ is determined as a function of the target false-alarm probability, to ensure "constant falsealarm rate (CFAR)" detection. The corresponding detection probability (or the missed-detection probability $P_{\mathrm{md}}=1-P_{\mathrm{d}}$ ) is also very important for CR networks where the interference caused by an opportunistic user to primary users must be very limited. As an example, the requirements imposed by the WRAN standard [3] are $P_{\mathrm{fa}}<0.1$ and $P_{\mathrm{md}}<0.1$.

\section{Considered Methods}

We restrict our analysis to non-parametric detection methods, i.e., which do not assume any prior knowledge about the signal to be detected. We focus on the difference in detection performance between the cases of known and unknown noise level $\left(\sigma_{v}^{2}\right)$.

\section{A. Known Noise Variance}

When testing a simple hypothesis $\mathcal{H}_{0}$ against a simple alternative $\mathcal{H}_{1}$, in general, the most powerful test is given by the Neyman-Pearson (NP) likelihood ratio [15]. In the considered scenario, with unknown channel vector $\boldsymbol{h}$, the eigenvalues of the sample covariance matrix $\boldsymbol{R}$ are sufficient statistics for the NP test (see [16]-p.11, and [17]-Sec.III-A), which can be written as

$$
\mathrm{LRT}=\frac{p\left(\lambda_{1}, \cdots, \lambda_{K} \mid \mathcal{H}_{1}\right)}{p\left(\lambda_{1}, \cdots, \lambda_{K} \mid \mathcal{H}_{0}\right)} .
$$

In the asymptotical regime $N \rightarrow \infty$, with given signal strength $\rho$ and noise variance $\sigma_{v}^{2}$, the above criterion can be shown [16], [17] to depend only on the largest eigenvalue $\left(\lambda_{1}\right)$, i.e., it reduces to Roy's largest root test [11], defined as

$$
T_{\mathrm{RLRT}} \triangleq \frac{\lambda_{1}}{\sigma_{v}^{2}}
$$

Notice that energy detection (ED), another commonly used criterion for known noise level, is suboptimal to RLRT in the sense of the Neyman-Pearson lemma. It can be written as

$$
T_{\mathrm{ED}} \triangleq \frac{1}{K N \sigma_{v}^{2}} \sum_{k=1}^{K} \sum_{n=1}^{N}\left|y_{k}(n)\right|^{2}=\frac{\|\boldsymbol{Y}\|_{F}^{2}}{K N \sigma_{v}^{2}}
$$

where $\|\cdot\|_{F}$ denotes the Frobenius norm. Since $\|\boldsymbol{Y}\|_{F}^{2}=$ $\operatorname{tr}\left(\boldsymbol{Y} \boldsymbol{Y}^{H}\right)$, we obtain that $T_{\mathrm{ED}}=\frac{1}{K \sigma^{2}} \sum_{i=1}^{K} \lambda_{i}$. Therefore, asymptotically in $N$, ED has reduced statistical power compared to (9) as it tests against the noise level the sum of all eigenvalues, instead of just $\lambda_{1}$.

\section{B. Unknown Noise Variance}

When $\sigma_{v}^{2}$ is unknown, $\mathcal{H}_{0}$ and $\mathcal{H}_{1}$ are composite hypothesis and the NP lemma does not apply. A common procedure is the GLRT, obtained from

$$
\text { GLRT }=\frac{\sup _{\boldsymbol{h}, \sigma_{v}^{2}} p\left(\boldsymbol{Y} \mid \mathcal{H}_{1}\right)}{\sup _{\sigma_{v}^{2}} p\left(\boldsymbol{Y} \mid \mathcal{H}_{0}\right)},
$$

which in our model ${ }^{3}$ is equivalent to (see [8], Sec. II)

$$
T_{\mathrm{GLRT}} \triangleq \frac{\lambda_{1}}{\frac{1}{K} \operatorname{tr}(\boldsymbol{R})} \text {. }
$$

Note that, since

$$
\frac{1}{T_{\mathrm{GLRT}}}=\frac{\sum_{i=1}^{K} \lambda_{i}}{\lambda_{1}}=1+\frac{\sum_{i=2}^{K} \lambda_{i}}{\lambda_{1}},
$$

the GLRT is equivalent (up to a nonlinear monotonic transformation) to

$$
T_{\mathrm{GLRT}^{\prime}}=\frac{\lambda_{1}}{\frac{1}{K-1} \sum_{i=2}^{K} \lambda_{i}} .
$$

The denominator of $T_{\mathrm{GLRT}^{\prime}}$ is the maximum-likelihood (ML) estimate of the noise variance assuming the presence of a signal [18], hence the GLRT can be interpreted as a largest root test with an estimated $\hat{\sigma}_{v}^{2}$ instead of the true (unknown) $\sigma_{v}^{2}$.

Remark: another popular detection criterion, proposed in [5], is the "eigenvalue ratio test" $T_{\mathrm{ERD}}=\lambda_{1} / \lambda_{K}$ (also called maximum-minimum eigenvalue, or condition number test). Compared to (12), this test is clearly suboptimal unless $K=2$.

Based on the above considerations, RLRT and GLRT are taken as the reference detection methods for the cases of known and unknown noise variance, respectively.

\section{Detection Performance of RLRT And GLRT}

In [8] it is shown that, in the asymptotic regime $N, K \rightarrow \infty$ with $K / N$ fixed, the GLRT detection performance converges to that of RLRT. However, a natural question is: how different is their performance for realistic values of $K, N$ ? In other words, what is the performance gap gained in practical applications by exact knowledge of the noise level? To answer this

\footnotetext{
3 This expression of the GLRT is specific to the considered model (single signal, unknown $\sigma^{2}$, unknown channel $\boldsymbol{h}$ ), i.e., the same as in [8]. Other GLRTs for different models, e.g., unknown number of signals and generic signal covariance matrix, are derived in [10].
} 


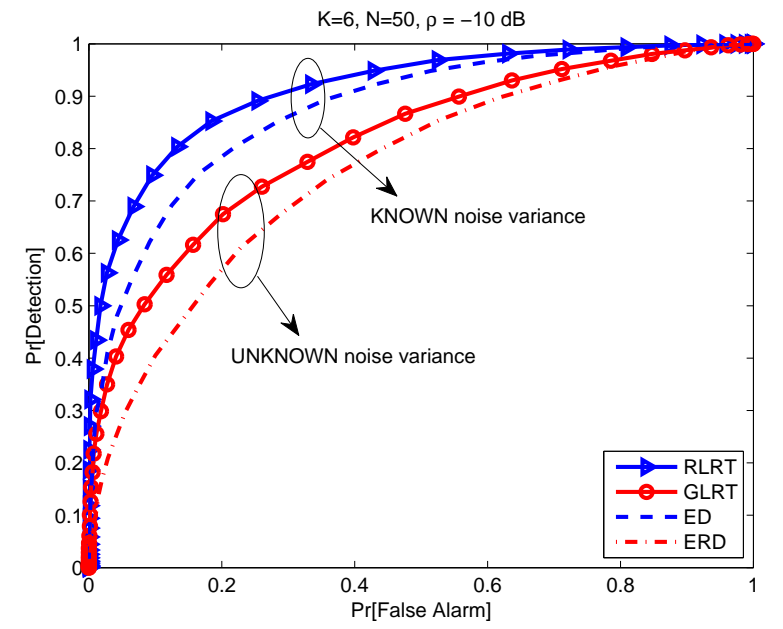

Fig. 1. Simulated receiver operating characteristics (ROC) curves for detection methods with known and unknown noise variance.

question, we compare the performance of the two detectors by deriving analytical expressions for the detection probability (7), given a false-alarm rate (6) $P_{\mathrm{fa}}=\alpha$ and a SNR $\rho$. Our goal is to express the detection probability (7) of the two methods,

$$
P_{\mathrm{d}}=\operatorname{Pr}\left(T>t(\alpha) \mid \mathcal{H}_{1}, \rho\right)
$$

where $t(\alpha)$ is the decision threshold such that $P_{\mathrm{fa}}=\alpha$. Since we are interested in low false alarm probabilities, we assume $\alpha \ll 1$.

A preliminary performance assessment is provided by Fig. 1, which compares the four aforementioned methods (RLRT, GLRT, ED and ERD) in a typical scenario for CR applications: small number of samples and receivers $(K=6, N=50)$ and a single signal with low SNR $(\rho=-10 \mathrm{~dB})$. These simulation results illustrate the performance gap between RLRT and GLRT, as well as the suboptimality of ED compared to RLRT (scenario of known noise level) and of ERD compared to GLRT (scenario of unknown noise level).

\section{A. RLRT}

1) Setting the threshold: For RLRT, the decision threshold $t(\alpha)$ can be approximated thanks to the property that under $\mathcal{H}_{0}$, and in the joint limit $N, K \rightarrow \infty$, the random variable $T_{\text {RLRT }}$ asymptotically follows a second-order Tracy-Widom (TW) distribution [19]:

$$
\operatorname{Pr}\left[\frac{T_{\mathrm{RLRT}}-\mu}{\xi}<s\right] \rightarrow F_{\mathrm{TW} 2}(s)
$$

with suitably chosen centering and scaling parameters

$$
\begin{aligned}
\mu & =\left[(K / N)^{1 / 2}+1\right]^{2} \\
\xi & =N^{-2 / 3}\left[(K / N)^{1 / 2}+1\right]\left[(K / N)^{-1 / 2}+1\right]^{1 / 3}
\end{aligned}
$$

(refined expressions of $\mu$ and $\xi$, providing an improved convergence rate, are given in [20]). Therefore,

$$
\begin{aligned}
\alpha & =\operatorname{Pr}\left(T_{\mathrm{RLRT}}>t\right)=\operatorname{Pr}\left(\frac{T_{\mathrm{RLRT}}-\mu}{\xi}>\frac{t-\mu}{\xi}\right) \\
& \approx 1-F_{\mathrm{TW} 2}\left(\frac{t-\mu}{\xi}\right) .
\end{aligned}
$$

Hence an approximate expression for the threshold of RLRT is

$$
t_{\mathrm{RLRT}}(\alpha) \approx \mu+F_{\mathrm{TW} 2}^{-1}(1-\alpha) \xi
$$

where $F_{\mathrm{TW} 2}^{-1}$ is the inverse of the TW c.d.f.

2) Detection probability: Under $\mathcal{H}_{1}$, the asymptotic distribution of $\lambda_{1}$ in the joint limit $N, K \rightarrow \infty$ is characterized by a phase transition phenomenon [21]. In the case of a single signal, the critical detection threshold for $N, K \rightarrow \infty$ can be expressed directly in terms of the SNR as [22]

$$
\rho_{\text {crit }}=\frac{1}{\sqrt{K N}} \text {. }
$$

This expression can be refined by adding correction terms for finite $N, K$ (cf. [17], Eq. 25). If the SNR $\rho$ is lower than the critical value, the limiting distribution of $\lambda_{1}$ under $\mathcal{H}_{1}$ is the same as that of the largest eigenvalue under $\mathcal{H}_{0}$, thus nullifying the statistical power of a largest eigenvalue test. If $\rho>\rho_{\text {crit }}$, on the contrary, the distribution of $T_{\text {RLRT }}$ is asymptotically Gaussian [17], [21], with

$$
\begin{aligned}
\mathrm{E}\left[\frac{\lambda_{1}}{\sigma_{v}^{2}}\right] & =(1+K \rho)\left(1+\frac{K-1}{N K \rho}\right) \\
\operatorname{Var}\left[\frac{\lambda_{1}}{\sigma_{v}^{2}}\right] & =\frac{1}{N}(K \rho+1)^{2},
\end{aligned}
$$

up to $O\left(1 / N^{2}\right)$ terms. Therefore, the detection probability can be expressed as

$$
P_{\mathrm{d}}^{(\mathrm{RLRT})} \approx Q\left[\sqrt{N}\left(\frac{t(\alpha)}{K \rho+1}-\frac{K-1}{N K \rho}-1\right)\right]
$$

where $Q(z)=\frac{1}{\sqrt{2 \pi}} \int_{z}^{\infty} e^{-x^{2} / 2} d x$ is the standard Gaussian tail probability function.

\section{B. GRLT}

1) Setting the threshold: Asymptotically, as both $N, K \rightarrow$ $\infty$, the random variable $T_{\mathrm{GLRT}}$ also follows a second-order TW distribution [8], hence in first approximation $t_{\mathrm{GLRT}}(\alpha) \approx$ $t_{\mathrm{RLRT}}(\alpha)$. However, as described in [23], this approximation is not very accurate for tail probabilities of $T_{\mathrm{GLRT}}$ for small values of $K$. In [23] the following improved expression was derived:

$\operatorname{Pr}\left[\frac{T_{\mathrm{GLRT}}-\mu}{\xi}<s\right] \approx F_{\mathrm{TW} 2}(s)-\frac{1}{2 N K}\left(\frac{\mu}{\xi}\right)^{2} F_{\mathrm{TW} 2}^{\prime \prime}(s)$.

Hence, for a required false alarm probability $\alpha$,

$$
\begin{aligned}
\alpha & =\operatorname{Pr}\left(T_{\mathrm{GLRT}}>t\right)=\operatorname{Pr}\left(\frac{T_{\mathrm{GLRT}}-\mu}{\xi}>\frac{t-\mu}{\xi}\right) \\
& \approx 1-F_{\mathrm{TW} 2}\left(\frac{t-\mu}{\xi}\right)+\frac{1}{2 N K}\left(\frac{\mu}{\xi}\right)^{2} F_{\mathrm{TW} 2}^{\prime \prime}\left(\frac{t-\mu}{\xi}\right) .
\end{aligned}
$$


The above equation can be numerically inverted to find the required threshold $t_{\mathrm{GLRT}}(\alpha)$.

2) Detection probability: To derive an explicit approximate expression for the detection performance of the GLRT under $\mathcal{H}_{1}$, we note that $\frac{1}{K} \sum_{j=1}^{K} \lambda_{j}=\frac{1}{K}\left[\lambda_{1}+\sum_{j=2}^{K-1} \lambda_{j}\right]$ and rewrite the GLRT (11) as

$$
\lambda_{1}>\tilde{t}(\alpha) \frac{\sum_{j=2}^{K} \lambda_{j}}{K-1}
$$

with

$$
\tilde{t}(\alpha)=\frac{K-1}{K-t_{\mathrm{GLRT}}(\alpha)} t_{\mathrm{GLRT}}(\alpha) .
$$

Assuming the presence of a sufficiently strong signal $(\rho>$ $\rho_{\text {crit }}$ ), the largest sample eigenvalue is (with high probability) due to a signal whereas the remaining eigenvalues, $\lambda_{2}, \ldots, \lambda_{K}$, are due to noise. Let

$$
Z \triangleq \frac{1}{K-1} \sum_{j=2}^{K} \lambda_{j}
$$

denote their mean. As discussed in [24](Eq.12), asymptotically in $N$, the random variable $Z$ is Gaussian distributed with variance $O\left(\frac{1}{N(K-1)}\right)$, and with a mean value that is slightly biased downwards:

$$
\mathrm{E}\left[\frac{Z}{\sigma_{v}^{2}}\right]=1-\frac{1}{N} \frac{K \rho+1}{K \rho}+O\left(\frac{1}{N^{2}}\right) .
$$

We then recall that $\lambda_{1} / \sigma_{v}^{2}$ is asymptotically Gaussian distributed with mean and variance given by (18) and (19). For a large number of sensors $(K \gg 1)$, the fluctuations of $Z$ are relatively much smaller than those of $\lambda_{1}$, hence we can approximate (22) as

$$
(1+K \rho)\left(1+\frac{K-1}{N K \rho}\right)+\frac{1+K \rho}{\sqrt{N}} \eta_{1}>\tilde{t}(\alpha) \cdot \mathrm{E}\left[\frac{Z}{\sigma_{v}^{2}}\right],
$$

where $\eta_{1} \sim \mathcal{N}(0,1)$ is a standard Gaussian random variable. Therefore, we conclude that

$P_{\mathrm{d}}^{(\mathrm{GLRT})} \approx Q\left[\sqrt{N}\left(\tilde{t}(\alpha)\left(\frac{1}{K \rho+1}-\frac{1}{N K \rho}\right)-\frac{K-1}{N K \rho}-1\right)\right]$.

\section{Performance Gap between RLRT and GRLT}

Comparison of (26) with (20) shows quantitatively the performance gain obtained using RLRT, i.e., knowing exactly the noise level, instead of GLRT, i.e., estimating the noise level from the sample eigenvalues. The difference in standard deviations between the detection probabilities in the two cases can be expressed, after some algebraic manipulations, as

$\frac{\sqrt{N}}{1+K \rho}\left(\frac{K-1}{K-t_{\mathrm{GLRT}}(\alpha)} t_{\mathrm{GLRT}}(\alpha)-t_{\mathrm{RLRT}}(\alpha)\right)+O\left(\frac{1}{\sqrt{N}}\right)$

This expression can be further simplified when $N \gg$ $(1 / K \rho)^{2}$. Under this condition, and recalling the expressions of $\mu$ (14) and $\xi(15)$, the threshold $t_{\mathrm{RLRT}}(\alpha)$ (16) can be written as

$$
t_{\mathrm{RLRT}}(\alpha)=1+2 \sqrt{\frac{K}{N}}+\frac{s_{\alpha}}{K^{1 / 6} \sqrt{N}}+O\left(\frac{1}{N}\right)
$$

where $s_{\alpha}=F_{\mathrm{TW} 2}^{-1}(1-\alpha)=O(1)$. A similar expression holds for $t_{\mathrm{GLRT}}(\alpha)$, with $s_{\alpha}$ replaced by $s_{\alpha, K, N}^{\prime}$, found by inverting (21) and, in general, also having a weak dependence on $N, K$. Inserting these expressions into (27) gives that, for $N \gg(1 / K \rho)^{2}$, the difference is roughly

$\frac{1}{1+K \rho}\left[\frac{2 \sqrt{K}}{K-1}+\frac{s_{\alpha, K, N}^{\prime}-s_{\alpha}}{K^{1 / 6}}+\frac{s_{\alpha, K, N}^{\prime}}{(K-1) K^{1 / 6}}\right]+O\left(\frac{1}{\sqrt{N}}\right)$.

We remark that for $\alpha \ll 1$, the difference $s_{\alpha, K, N}^{\prime}-s_{\alpha}$ is negative but quite small even for a small number of sensors. For large $N$ the first term is thus the dominant one. From this term we see that, as expected, the performance gap between Roy's largest root test and the GLRT decreases with a larger number of sensors, for which we have a better noise estimate, but at a relatively slow rate of $O(1 / \sqrt{K})$.

In particular, for a practical number of sensors, this gap is non-negligible. For example, for detection of weak signals $(K \rho \ll 1)$ with an array of $K=4$ sensors, at a fixed alarm of $\alpha=1 \%$, and over a wide range of values of $N$, the difference is about 1.4 standard deviations. Even with $K=16$ sensors, the difference is still about 0.5 standard deviations.

The performance gap between RLRT and GLRT can be evaluated also in terms of SNR needed by the two detectors to achieve the same $P_{\mathrm{d}}$. Let us set $P_{\mathrm{d}}^{(\mathrm{RLRT})}=P_{\mathrm{d}}^{(\mathrm{GLRT})}$; after some algebra and neglecting $O(1 / N)$ terms, we obtain

$$
\frac{\rho_{G L R T}}{\rho_{R L R T}} \approx \frac{\tilde{t}(\alpha)}{t_{\mathrm{RLRT}}(\alpha)}+\frac{1}{K \rho_{R L R T}} \frac{\tilde{t}(\alpha)-t_{\mathrm{RLRT}}(\alpha)}{t_{\mathrm{RLRT}}(\alpha)} .
$$

Now, from Eq. (23) it follows that $\tilde{t}(\alpha)>t_{\mathrm{RLRT}}(\alpha)$ : in fact, even though $t_{\mathrm{GLRT}}(\alpha)$ is slightly lower than $t_{\mathrm{RLRT}}(\alpha)$, the term $(K-1) /\left(K-t_{\mathrm{GLRT}}(\alpha)\right)>1$ is largely dominant for $\alpha \ll 1$. Therefore, $\rho_{\mathrm{GLRT}}>\rho_{\mathrm{RLRT}}$, i.e., RLRT achieves the same detection performance of the GLRT for signals with a lower SNR. Numerical examples are shown in next section.

\section{Simulation Results}

In Fig. 2 we compare the detection performance of RLRT and GLRT with the theoretical formulas (20) and (26), as a function of the SNR $\rho$ and for a given false alarm rate of $\alpha=0.5 \%$. Fig. 3 illustrates the SNR gap between GLRT and RLRT, i.e., the extra SNR needed by the GLRT to achieve the same detection probability of RLRT. The figure shows that the gap increases for (i) small number of sensors and (ii) low signal strength, in accordance to the theoretical formula (30). These conclusions highlight the key role that the noise variance estimation has for spectrum sensing, especially in challenging scenarios. 


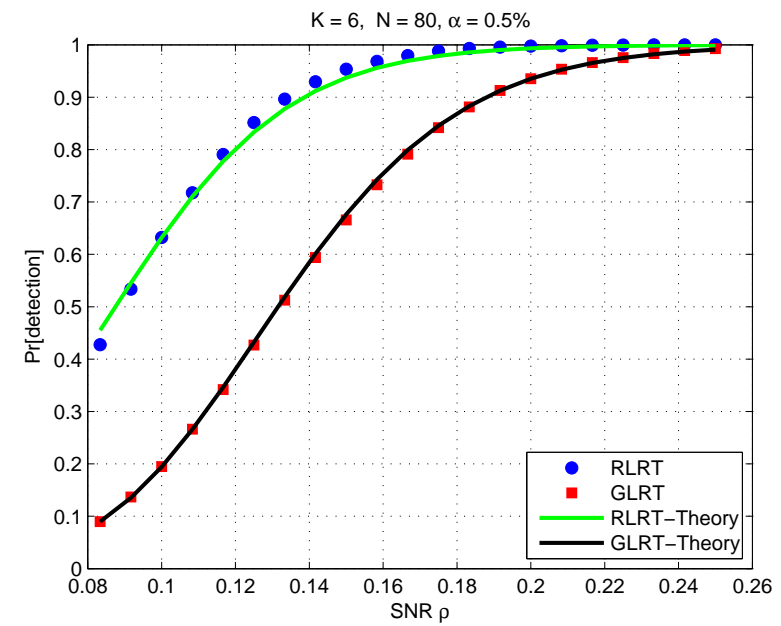

Fig. 2. Comparison of detection performance curves of RLRT and GLRT methods, from simulation and analytical expressions (20) and (26).

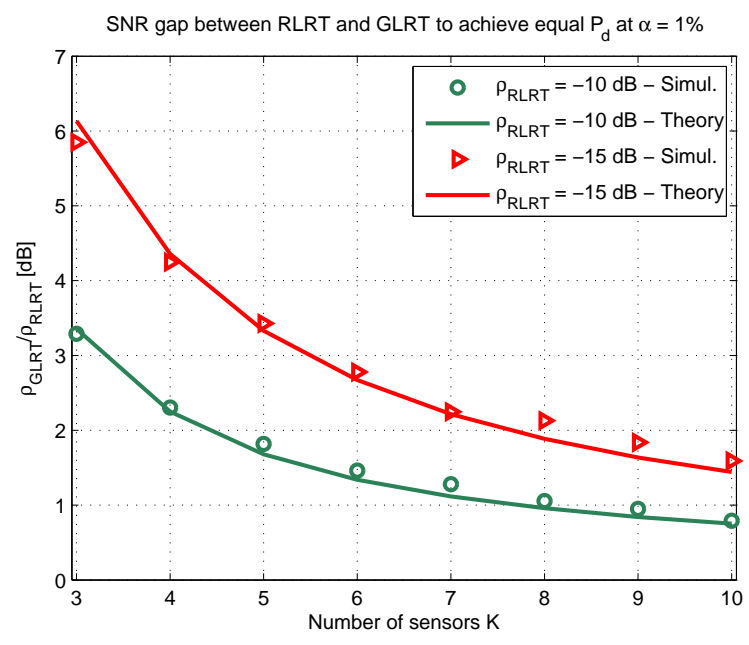

Fig. 3. SNR gap for GLRT to achieve the same $P_{\mathrm{d}}$ as RLRT, as a function of $K$ for different levels of signal strength $\left(\rho_{\mathrm{RLRT}}\right)$. Simulation results vs. analytical approximation (30). Fixed false alarm rate $\alpha=1 \%, N=100$.

\section{Conclusions}

The performance of eigenvalue-based detection has been investigated in this paper, considering conditions of known and unknown noise level. Closed-form expressions of detection probability of two best-performing detectors in their respective classes have been derived. These results provide a performance evaluation of two important detection techniques in cognitive radio systems, and can be used for an accurate design of spectrum sensing parameters (number of sensors and samples) given a target false alarm/detection rate imposed by standards (e.g., $P_{\mathrm{d}}=0.9$ at $\mathrm{SNR}=-10 \mathrm{~dB}$ with $P_{\mathrm{fa}}=0.1$ ). In addition, the quantitative characterization of the gap between GLRT and RLRT gives insight into the impact of knowing the exact noise level, which results in a significant performance improvement especially when the number of sensors is low.

Based on this analysis, further research will be carried out towards "hybrid" detection methods where auxiliary time slots may be used to obtain a refined noise variance estimate and thus reduce the gap between GLRT and RLRT.

\section{REFERENCES}

[1] J. Mitola and G. Q. Maguire, "Cognitive radios: making software radios more personal", IEEE Personal Commun., vol. 6, no. 4, pp. 13-18, 1999.

[2] S. Haykin, "Cognitive radio: brain-empowered wireless communications", IEEE Trans. Commun., vol. 23, no. 2, pp. 201-220, 2005.

[3] IEEE 802.22, "Draft Standard for Wireless Regional Area Networks Part 22" July 2008.

[4] Y. Zeng, Y.-C. Liang, A. T. Hoang, and R. Zhang, "A Review on Spectrum Sensing for Cognitive Radio: Challenges and Solutions", EURASIP Journal on Advances in Signal Processing, vol. 2010, pp. 1-15, January 2010.

[5] Y. H. Zeng and Y.-C. Liang, "Eigenvalue based spectrum sensing algorithms for cognitive radio", IEEE Trans. on Communications, vol. 57, no. 6, pp. 1784-1793, June 2009.

[6] F. Penna, R. Garello, M. A. Spirito, "Cooperative Spectrum Sensing based on the Limiting Eigenvalue Ratio Distribution in Wishart Matrices", IEEE Comm. Letters, vol.13, no.7, pp.507-509, July 2009.

[7] P. Bianchi, J. Najim, G. Alfano, and M. Debbah, "Asymptotics of eigenbased collaborative sensing", Proc. IEEE Information Theory Workshop (ITW 2009), Taormina, Italy, Oct. 2009.

[8] P. Bianchi, M. Debbah, M. Maida, and J. Najim, "Performance of Statistical Tests for Source Detection using Random Matrix Theory", http://arxiv.org/abs/0910.0827.

[9] Y. Zeng, Y.C. Liang, R. Zhang, "Blindly combined energy detection for spectrum sensing in cognitive radio", IEEE Signal Processing Letters, vol. 15, 2008.

[10] R. Zhang, T.J. Lim, Y.C. Liang, Y. Zeng, "Multi-antenna based spectrum sensing for cognitive radios: a GLRT approach", IEEE Trans. Communications, vol. 58, no. 1, Jan. 2010.

[11] S. N. Roy, "On a heuristic method of test construction and its use in multivariate analysis", Ann. Math. Stat., vol. 24, no. 2, pp. 220-238, 1953.

[12] O. Besson, L.L. Scharf, "CFAR matched direction detector", IEEE Trans. on Sig. Proc., vol.54, no.7, pp.2840-2844, July 2006.

[13] D. E. Johnson, F. A. Graybill, "An analysis of a two-way model with interaction and no replication", J. Amer. Stat. Assoc. no. 67, pp. 862-868, 1972.

[14] J. Schott, "A note on the critical values used in stepwise tests for multiplicative components of interaction", Comm. Stat. Th. Meth., vol 15, no. 5, pp. 1561-1570, 1986.

[15] J. Neyman, E. Pearson, "On the Problem of the Most Efficient Tests of Statistical Hypotheses", Philosophical Transactions of the Royal Society of London, Series A, 231: 289-337, 1933.

[16] R. J. Muirhead, "Latent roots and matrix variates: A review of some asymptotic results," Ann. Stat., vol. 6, no. 1, pp. 5-33, 1978.

[17] S. Kritchman, B. Nadler, "Non-Parametric Detections of the Number of Signals: Hypothesis Testing and Random Matrix Theory", IEEE Trans. on Signal Processing, vol. 57, no. 10, pp. 3930-3941, 2009.

[18] M. Wax and T. Kailath, "Detection of Signals by Information Theoretic Criteria", IEEE Trans. on Acoustics, Speech and Signal Processing, vol. ASSP-33, no. 2, pp.387-392, Apr. 1985.

[19] I. M. Johnstone, "On the distribution of the largest eigenvalue in principal component analysis", Annals of Statistics, vol.29, no.2, pp.295327, 2001

[20] N. El Karoui, "A rate of convergence result for the largest eigenvalue of complex white Wishart matrices", Ann. Prob., vol. 36, no. 6, pp. 20772117, 2006.

[21] J. Baik and J.W. Silverstein, "Eigenvalues of large sample covariance matrices of spiked population models", J. Mult. Anal., vol. 97, no 6, pp. 1382-1408, 2006

[22] F. Penna, R. Garello, M. A. Spirito, "Probability of Missed Detection in Eigenvalue Ratio Spectrum Sensing", Proc. 5th IEEE Int. Conf. on Wireless and Mobile Computing, Networking and Communications (WiMob), Marrakech, Morocco, Oct. 2009

[23] B. Nadler, On the distribution of the ratio of the largest eigenvalue to the trace of a Wishart Matrix, J. of Mult. Anal., vol. 102, pp. 363-371, 2010.

[24] S. Kritchman and B. Nadler, "Determining the number of components in a factor model from limited noisy data", Chemometrics and Intelligent Laboratory Systems, vol. 94, pp. 19-32, 2008. 